\title{
Unstable genomes promote inflammation
}

Faulty DNA replication can make genomes unstable. It now seems that, in mice, severe disruption of DNA replication triggers inflammation in the placenta, and female embryos are more sensitive to this than are male ones. SEE LETTER P.105

\section{SARAH LAMBERT}

$\mathrm{T}$ The development of an embryo requires thousands of rapid cell divisions, during each of which the entire genome is replicated. Perturbations in the process of DNA replication can generate an unstable genome. McNairn et al. ${ }^{1}$ reveal on page 105 that, in mice, genomic instability caused by defective DNA replication during embryonic development triggers an inflammatory response that preferentially kills female embryos. This sexbiased embryonic death is caused by the inability of female embryos to produce testosterone, an anti-inflammatory hormone. The authors' findings have implications for cancer biology.

During cell division, chromosomes are duplicated and then segregated evenly to each daughter cell. DNA replication is the process whereby each nucleotide - DNA building block - of each chromosome is duplicated by DNA synthesis. The double-stranded DNA of the parent cell is unwound at thousands of sites called replication origins, to allow each strand to be duplicated through the sustained action of DNA-protein structures called replication forks.

All replication origins are bound by macromolecular complexes that enable (license) DNA synthesis, but this process occurs only at some of them ${ }^{2}$. The others, called dormant origins, act as a back-up for when DNA synthesis at replication forks is inefficient. The activation of dormant origins creates extra initiation events to sustain the complete duplication of chromosomes before segregation.

The minichromosome maintenance complex (MCM), which is composed of six different protein subunits, is part of the machinery responsible for opening doublestranded DNA at replication origins and during the progression of replication forks ${ }^{2}$ (Fig. 1). A decrease in the amount of MCM in cells reduces the total number of initiation events and the ability of dormant origins to back up the completion of DNA replication $^{3}$. Previous work from the current paper's research group showed that a $40 \%$ reduction in the amount of MCM in cells causes genomic instability in mice, increases the likelihood of death before or after birth ${ }^{4}$, and, in some cases, favours the development of cancer ${ }^{5}$.

McNairn et al. report that female embryos with MCM deficiency are two to three times more likely to die than are their male counterparts. This female-biased death occurred after the stage of sex determination, when secondary sexual characteristics appear. Anti-inflammatory treatments, such as ibuprofen or testosterone, protected the female embryos from death without restoring MCM function. Conversely, both female and male embryos died when the receptor for the naturally occurring anti-inflammatory molecule IL-10 was inactivated. The authors propose that both female and male embryos with MCM deficiency have unstable genomes, but that female embryos are more sensitive to the inflammation that results from this instability (Fig. 1).

Genomic instability activates signalling pathways involved in inflammation ${ }^{6}$, innate immunity $^{7}$ and normal, programmed cell death ${ }^{7}$. Because each mouse embryo is embedded in its own placenta and a pregnant mouse carries many embryos, the authors could analyse the genetic make-up (genotype) of diverse combinations of maternal and embryonic cells. They saw that placental cells with MCMdependent genomic instability had high levels of DNA damage, regardless of the genotype of the embryo. Surprisingly, the authors detected active inflammation and innate-immune responses in placentae of female embryos with MCM-dependent genomic instability. Antiinflammatory treatment did not reduce DNA damage in placental cells. These observations indicate that DNA damage precedes, and can occur without, inflammation.

McNairn and colleagues' findings suggest that the maternal genotype influences the likelihood of female-embryo survival. Inflammation - and, therefore, sex-biased embryo death - occurs only when maternal and fetal genomic instability act in combination. A successful pregnancy requires an immunetolerant environment and the suppression of inflammation at the maternal-fetal interface.

A similar interaction between 'self' and 'non-self' exists in cancer, because tumours express proteins that are not found in normal cells. The interface between cancer cells and the tumour microenvironment recapitulates some aspects of the placental-embryo interface, including high levels of cell proliferation and invasion and suppression of the immune

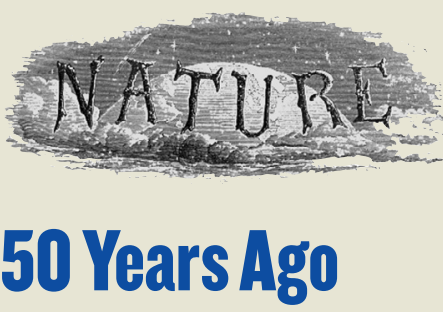

Musicians apprehensive about the advance of computers into their domain would have been well advised to avoid the Royal Institution on February 28. Professor C. A. Taylor of the University of Wales ... gave a discourse on "Physics and Music" which demonstrated the full potentialities of computer generated harmonics. One piece which particularly caught the audience's fancy was a sequence of notes which appeared to be a chromatic scale, but which never appeared to increase in pitch. Professor Taylor explained that this was achieved by adding together several harmonics of a basic note and then modulating these with an intensity function in the shape of an inverted letter U. By keeping the centre of the $U$ at the same pitch while increasing the individual notes chromatically, the mind is led to experience the dual sensation of pitch stability and increase.

From Nature 8 March 1969

100 Years Ago

Mr. J. Reid Moir describes ... a remarkable piece of carved chalk recently found ... at ...Suffolk. The specimen ... is of a dull white colour, and has sandy material embedded in the interstices. It is believed that it was brought to the surface by the action of rabbits ... Mr. Moir believes that ... the piece of chalk bears a very close resemblance to the outline of the mammoth (E. primigenesis), with which the scientific world has become familiar by an examination of carcasses of this animal found in the frozen ground of Siberia ... The specimen certainly exhibits many remarkable points of resemblance to the mammoth, but the question remains whether these resemblances may not be accidental or the result of weathering. It may be advisable to await further examination by experts before we express a decided opinion upon this remarkable discovery. From Nature 6 March 1919 